Article

\title{
Ansamitocin P3-Loaded Gold-NanoCage Conjugated with Immune Checkpoint Inhibitor to Enhance Photo-Chemo-Thermal Maturation of Dendritic Cells for Hepatocellular Carcinoma
}

\author{
Hung-Wei Cheng ${ }^{1}$, Yu-Ling Ou ${ }^{1}$, Chia-Chi Kuo ${ }^{1}$, Hsin-Yi Tsao ${ }^{1}$ and Huai-En Lu ${ }^{2, *}$ \\ 1 Department of Materials Science and Engineering, National Yang Ming Chiao Tung University, \\ Hsinchu 30010, Taiwan; mlb14756@gmail.com (H.-W.C.); annou831101@yahoo.com.tw (Y.-L.O.); \\ d42172000@gmail.com (C.-C.K.); yvone880217@gmail.com (H.-Y.T.) \\ 2 Bioresource Collection and Research Center, Food Industry Research and Development Institute, \\ Hsinchu 300193, Taiwan \\ * Correspondence: hel@firdi.org.tw
}

check for

updates

Citation: Cheng, H.-W.; Ou, Y.-L.; Kuo, C.-C.; Tsao, H.-Y.; Lu, H.-E.

Ansamitocin P3-Loaded

Gold-NanoCage Conjugated with

Immune Checkpoint Inhibitor to

Enhance Photo-Chemo-Thermal Maturation of Dendritic Cells for Hepatocellular Carcinoma. Polymers 2021, 13, 2726. https://doi.org/ $10.3390 /$ polym 13162726

Academic Editor: Gerald Guerin

Received: 6 July 2021

Accepted: 10 August 2021

Published: 15 August 2021

Publisher's Note: MDPI stays neutra with regard to jurisdictional claims in published maps and institutional affiliations.

Copyright: (c) 2021 by the authors. Licensee MDPI, Basel, Switzerland. This article is an open access article distributed under the terms and conditions of the Creative Commons Attribution (CC BY) license (https:// creativecommons.org/licenses/by/ $4.0 /)$.

\begin{abstract}
Immunotherapy is a newly developed method for cancer treatment, but still generates limited response in partial patients for hepatocellular carcinoma (HCC) because the immunity cycle is limited by the tumor microenvironment (TME). Herein, we introduce multifunctional gold nanocages (AuNCs)-based nanocarriers with Ansamitocin P3 (AP3) loaded and anti-PDL1 binding (AP3AuNCs-anti-PDL1) which can combine photothermal therapy, chemotherapeutic agent-triggered DCs maturation, and checkpoint immunotherapy in one platform. The AP3-AuNCs-anti-PDL1 using Avidin-biotin to bind anti-PDL1 on the surface of AP3-AuNCs showed specifically cellular targeting compared to AuNCs, which can increase the immune responses. The AP3-AuNCs+NIR-10 min exhibited the highly activated DCs maturation with two-fold higher than control+NIR, which can be attributed to the significant release of AP3. The results illustrated the synergistic effect of tumor-associated antigens (TAAs) and controlled AP3 release under near infrared (NIR) in triggering effective DCs maturation. Among them, AP3 release played the more important role than the TAAs under PTT in promoting T-cell activation. These results illustrate the promising potential of AuNCsbased nanocarriers combined with AP3 and the checkpoint inhibitors to strengthen the positive loop of immunity cycle.
\end{abstract}

Keywords: gold nanocages; photothermal therapy; DCs maturation; immunotherapy; immune checkpoint inhibitor

\section{Introduction}

Immunotherapy has become a promising therapeutic strategy for treating cancer. Many immune treatments have now been developed to fight cancer via stimulating immune system to attack cancer cells, but currently a few trials are conducted for hepatocellular carcinoma (HCC) because of low patient response rate of immune checkpoint inhibitors (ICIs) which are associated with decreased tumor infiltration of CD8+ T cells and block dendritic cells (DCs) function in non-immunogenic tumor microenvironment [1-8]. To achieve effective immunotherapy, a series of functional stepwise pathways called the "cancer-immunity cycle" where the tumor-associated antigens (TAAs) presented on DCs surface has to be initiated [9]. There are many methods to induce the formation of TAAs by the immunogenic cell death (ICD), such as chemotherapy, radiation therapy, and photothermal therapy (PTT). Further, ICD can express the damage-associated molecular patterns (DAMPs) to stimulate DCs maturation and migration [10,11]. Among them, PTT was usually considered as the best candidate to selectively heat tumor region without damaging the normal cells compared to other treatments $[12,13]$. However, the maturation on the 
DCs through TAAs induced by PTT may be limited to achieving effective activation of T cells since the inhibition of tumor microenvironment (TME) and localized near infrared (NIR), which need to add inducer of DCs stimulation for converting immature DCs into mature DCs [14-16]. Therefore, it became an important issue to develop a nanocarrier with both chemotherapy and photothermal therapy contributing to mature DCs for increasing tumor infiltration of CD8+ T cells to raise the response of ICIs.

Several drugs or strategies have been adopted to mature DCs [17-19]. Recently, lowdose (noncytotoxic concentrations) chemotherapeutic agents as immune-adjuvant were reported to induce DCs maturation. Martin et al. further investigated the pharmacological effect of different class cytotoxic compounds, and found that microtubule-destabilizing compounds were able to induce DCs stimulation [20]. Among them, Ansamitocin P3 (AP3), a kind of microtubule-depolymerizing agent isolated from Nocardia, was identified as the most effective inducer of DCs maturation to induce a potent phenotypic and functional maturation for activating T cells at a lower dose. In addition, AP3 can enhance T-cell stimulatory capacity through up-regulation of maturation markers on the DCs and production of pro-inflammatory cytokines [20]. However, AP3 is hardly soluble in water because its solubility in pure water is only $20 \mathrm{mg} / \mathrm{L}$ under normal temperature and pressure. Therefore, it becomes an important issue to develop a nanostructure as a platform to integrate the AP3 and the PTT with ICI addition for improving the immune response.

Gold $(\mathrm{Au})$ nanostructures have received wide attention because of their excellent optical properties, bio-inertness, and low cytotoxicity among all kinds of biocompatible nanoparticles [21-23]. In the study, to achieve both chemotherapy and PTT with enhanced permeability to spread and to eradicate wide-ranged malignant cells, a multifunctional AP3 loaded-AuNCs with anti-PDL1 targeting system (AP3-AuNCs-anti-PDL1) was designed to combine PTT, chemotherapeutic agent, and checkpoint immune therapy in one platform as shown in Scheme 1a. Under NIR irradiation at $808 \mathrm{~nm}$, the AP3 loaded-AuNCs are used as photosensitizer to produce PTT for generating tumor-associated antigen and simultaneously release AP3 to stimulate maturation of DCs to boost the immune response. In addition, anti-PDL1 checkpoint inhibitor can be used to selectively target the tumor cell which shows high expression of PDL1 on the surface to enhance the accuracy of PTT. The mechanisms and the advantages of the AP3-AuNCs-anti-PDL1 are also illustrated in Scheme 1b. First, anti-PDL1 is capable of blocking PDL1 on the surface of liver cancer cells (Hep55.1c) to increase the accuracy of PTT and enhance immune response. Second, PTT produced by AuNCs under the NIR exposure can not only induce apoptosis but also release AP3 at the same time to promote DCs maturation. Overall, this AuNCs-based nanocarrier with the anti-PDL1 as a platform to produce the TAAs and control the AP3 release for stimulating DCs maturation, lead to the activation of $\mathrm{T}$ cell and specific targeting of the tumor cells to create an immunogenic TME. The AP3-AuNCs-anti-PDL1 with targeting and PTT can achieve multiple stimuli to maturate the DCs, and thus the response rate of immune checkpoint inhibitors can be much enhanced. This strategy can provide more effective DCs maturation to increase the immuno-therapeutic efficacy and is worth further investigation to explore the actual in vivo effect in different tumor models. 
(a)
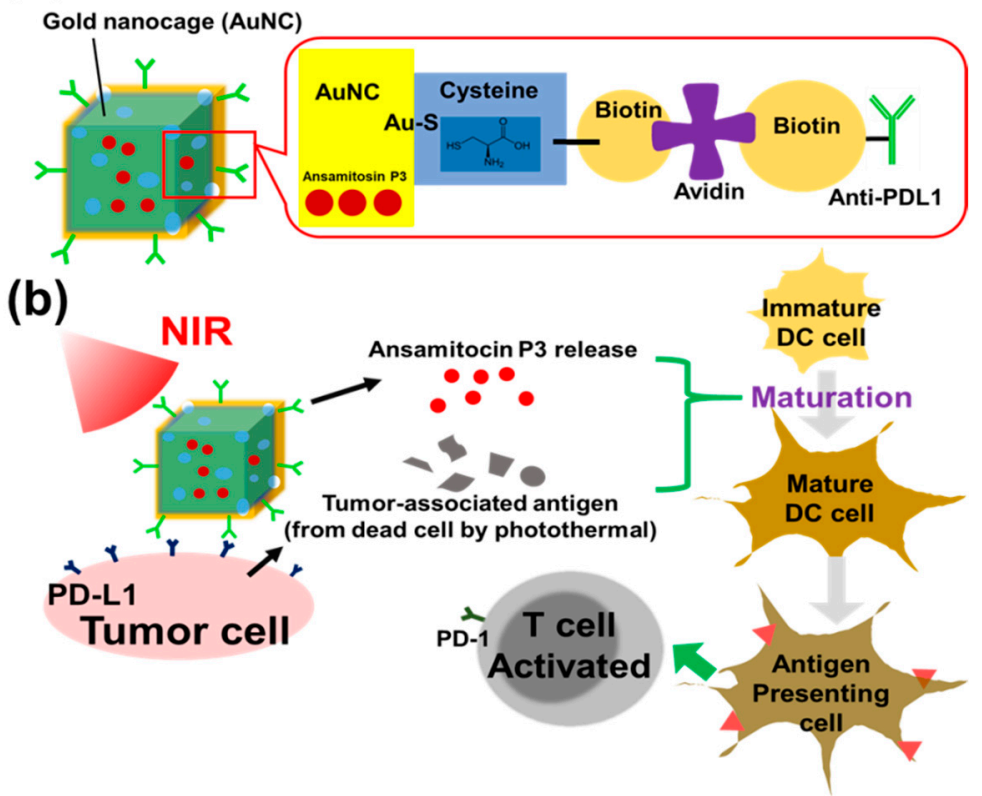

Scheme 1. Schematic illustration of (a) component of multifunctional AuNCs-based immunanoparticle and (b) the synergistic mechanisms of both photo-chemotherapy and photo-thermal therapy to mature DCs under NIR irradiation.

\section{Materials and Methods}

\subsection{Materials}

Ethylene glycol, silver nitrate $\left(\mathrm{AgNO}_{3}\right), \mathrm{PVP}$ (powder, average $\left.\mathrm{Mw} \sim 29,000\right), \mathrm{Na}_{2} \mathrm{~S}$ $9 \mathrm{H}_{2} \mathrm{O}, \mathrm{HAuCl}_{4} \cdot 3 \mathrm{H}_{2} \mathrm{O}$, sodium chloride, $\mathrm{N}$-hydroxysuccinimide (NHS, $98 \%$ ), $\mathrm{N}$-(3-dimethyl aminopropyl)-N'-ethylcarbodiimide hydrochloride (EDC, 98\%), L-cysteiene (CYS), ansamitocin P3 were purchased from Sigma Aldrich Chemical Co (Burlington, NJ, USA). Rhodamine $B$ used to label the nanocarriers for visualization under a fluorescence microscope was purchased from Ocean NanoTech (SAN DIEGO, CA, USA). Anti-PDL1 antibody, 4',6diamidino-2-phenylindole (DAPI), phalloidin, Rat IgG antibody (FITC) were purchased from Thermo Fisher Scientific (Waltham, MA, USA). Interleukin 4 (IL-4) was purchased from Sino (Wayne, MI, USA). Alexa Fluor ${ }^{\circledR} 488$ Goat Anti-Mouse IgG, F(ab')2 fragment specific, was purchased from Jackson ImmunoResearch (West Grove, PA, USA).

\subsection{Methods}

\subsubsection{Preparation of Gold Nanocages (Auncs)}

The procedure of synthesizing AuNCs followed Xia's group publication reported in 2007 [24]. The entire process was divided into two parts including synthesis of sliver nanocubes (AgNCs) and AuNCs. For AgNCs formation, briefly, the oil bath was pre-heated to $150^{\circ} \mathrm{C}$ and then four clean reaction vials containing ethylene glycol (EG, $6 \mathrm{~mL}$ ) were put into the oil bath for $1 \mathrm{~h}$. After that, two $\mathrm{Na}_{2} \mathrm{~S}$ EG solutions $(3 \mathrm{mM}, 70 \mu \mathrm{L}$, and $100 \mu \mathrm{L}$ ) were pipetted into the vials, respectively, followed by waiting for $8 \mathrm{~min}$. Then PVP EG solution $(0.18 \mathrm{M}, 1.5 \mathrm{~mL})$ and $\mathrm{AgNO}_{3}$ EG solution $(0.28 \mathrm{M}, 0.5 \mathrm{~mL})$ were pipetted into the four vials, followed by waiting for $15 \mathrm{~min}$. After the reaction vials cooled down to room temperature, the products were washed using acetone and spun down at $2000 \mathrm{~g}$ for $30 \mathrm{~min}$. Then, the products were again washed with deionized water for three times and centrifuged at $9000 \times g$ for $10 \mathrm{~min}$. AgNCs were dispersed in deionized (DI) water $(4 \mathrm{~mL})$ and stored at $4{ }^{\circ} \mathrm{C}$ until use. For synthesis of AuNCs, AgNCs solution and PVP solution $(9 \mathrm{mM}, 10 \mathrm{~mL})$ were pipetted into a round-bottom flask and then titrated $\mathrm{HAuCl}_{4}$ solution $(0.2 \mathrm{mM})$ was added to the mixture at a rate of $10-15 \mathrm{~mL} / \mathrm{h}$. For a desirable LSPR peak of AuNCs, color changes and UV-visible spectrum (Evolution 300, Thermo Fisher Scientific, 
Waltham, MA, USA) were both observed. After the reaction was stopped, excessive sodium chloride $(\mathrm{NaCl})$ was added to the mixture, and then the supernatant was collected and washed three times at $11,000 \mathrm{rpm}, 10 \mathrm{~min}$. AuNCs were collected in DI water at $4{ }^{\circ} \mathrm{C}$.

\subsubsection{Synthesis of Cys-Biotin-Avidin-Biotin-Anti-PDL1}

The synthesis process of L-cysteine (Cys)-Biotin-Avidin-Biotin-anti-PDL1 can be divided into two pathways. First, Cys-Biotin and Biotin-anti-PDL1 were synthesized. The Sulfo-NHS-Biotin (10 mM, $25 \mu \mathrm{L})$ was added to Cys $(1 \mathrm{mg} / \mathrm{mL})$ and anti-PDL1 $(1 \mathrm{mg} / \mathrm{mL})$ in the ice for $2 \mathrm{~h}$ in the dark, respectively. To purify the product, Cys-Biotin and anti-PDL1Biotin were centrifuged at $1000 \mathrm{rcf}$. for $2 \mathrm{~min}$ and repeated three times. The obtained Cys-Biotin-Avidin-Biotin-anti-PDL1 was suspended in deionized water at $4{ }^{\circ} \mathrm{C}$.

\subsubsection{Synthesis of AP3-Auncs-Cys-Biotin-Avidin-Biotin-Anti-PDL1} (AP3-AuNCs-anti-PDL1)

Both the Ansamitocin P3 (AP3) ethanol (EtOH) solution (50 ppm, $120 \mu \mathrm{L})$ and the CysBiotin-Avidin-Biotin-anti-PDL1 (1000 ppm, $9 \mu \mathrm{L})$ were added to $3 \mathrm{~mL}$ AuNCs $(50 \mu \mathrm{g} / \mathrm{mL})$. The mixed solution was reacted for $12 \mathrm{~h}$ at $4{ }^{\circ} \mathrm{C}$. To remove the unloaded AP3 and CysBiotin-Avidin-Biotin-anti-PDL1, the mixed solution was added to a dialysis tubing cellulose membrane with cut off molecule weight of 14,000. The dialysis bags were dialyzed in $6 \mathrm{~mL}$ DI water at room temperature for $30 \mathrm{~min}$. Then the DI water was changed and dialyzing was repeated four times. The obtained AP3-AuNCs-anti-PDL1 was suspended in DI water at $4{ }^{\circ} \mathrm{C}$.

\subsubsection{Characterization of AP3-Auncs-Anti-PDL1}

The morphology of AgNCs and AuNCs were characterized using scanning electron microscopy (SEM; JSM-6700-F, JEOL, Tokyo, Japan) and transmission electron microscopy (TEM; JSM-2100, JEOL, Tokyo, Japan). For SEM observation, the sample solution was dropped on silicon wafers, and dried in a vacuum desiccator at room temperature. For TEM observation, the sample solution was dropped on a carbon-coated copper grid. Residues of the droplet were removed and then the copper grid was dried in a vacuum desiccator at room temperature. The zeta potential and particle size of nanocages in DI water, $80 \% \mathrm{EtOH}$, and $99 \% \mathrm{EtOH}$ solvent were measured by electrophoretic light scattering and dynamic light scattering (ELS and DLS; Delsa Nano C particle analyzer, Beckman Coulter, Brea, CA, USA). In order to prove the AP3 were successfully loaded in the AuNCs at different solvents, the chemical state was analyzed by using Fourier transform infrared spectroscopy (FTIR, spectrum 100, PerkinElmer). To confirm the conjugation of anti-PDL1, Alexa Fluor ${ }^{\circledR} 488$ Goat Anti-Mouse IgG, F(ab')2 fragment specific was added to the AP3-AuNCs-anti-PDL1 for $1 \mathrm{~h}$ and washed three times. The results were observed using fluorescence microscopy (D-eclipse C1; Eclipse, TE2000-U, Nikon, Tokyo, Japan).

\subsubsection{Photothermic Effect of Aqueous AP3-Auncs}

The temperature of AP3-AuNCs solution (AuNCs, $50 \mu \mathrm{g} / \mathrm{mL}$ ) under $808 \mathrm{~nm}$ laser exposures $\left(1 \mathrm{~W} / \mathrm{cm}^{2}\right)$ and the temperature elevation plot was recorded by a thermal couple which operated at $30 \mathrm{~s}$ interval for a 15 -min period. The photothermal conversion efficiency (PTCE) of the AuNCs and AP3-AuNCs was determined according to the following formula (Equation (1)) [25,26]:

$$
\eta=\frac{h s\left(T_{M a x}-T_{\text {Surr }}\right)-Q_{D i s}}{I\left(1-10^{-A 808}\right)}
$$

where $h$ is the heat transfer coefficient, $s$ is the surface area of the container. $T_{M a x}-T_{\text {Surr }}$ is the temperature change of the AP3-AuNCs solution at the maximum steady environmental temperature, $I$ is the power of the laser, A808 is the absorbance of AP3-AuNCs at $808 \mathrm{~nm}$, and $Q_{D i s}$ expresses heat dissipated from light absorbed by the solvent and the container. 


\subsubsection{Drug-Loading Ability and in Vitro Drug Release}

Loading capacity and encapsulation efficiency (EE) of AP3-AuNCs were determined using liquid chromatography-mass spectrometry (LC-MS) [27]. In brief, after dialysis of the AP3-AuNCs, the DI water out of the dialysis bags was collected and the AP3 of the DI water was quantified using LC-MS. The loading capacity and EE of the Ansamitocin P3 in the AP3-AuNCs were calculated as follows (Equations (2) and (3)):

$$
\text { Loading capacity }\left(\frac{w}{w}\right)=\frac{A}{B}=\frac{D-C}{B}
$$

where $A$ is the mass of the carried drug, $B$ is the mass of the entire AP3-AuNCs, $C$ is the mass of the drug not carried by AP3-AuNCs and quantified by LC-MS, $D$ is the total mass of the added drug, and

$$
E E \%=\frac{E}{F} \times 100 \%=\frac{F-G}{F} \times 100 \%
$$

where $E$ is the amount of carried drug, $F$ is the total amount of drug in feeding formula, and $G$ is the amount of drug which stayed in solvent out of the dialysis bags and quantified by LC-MS.

For the drug release test, AP3-AuNCs $(50 \mu \mathrm{g} / \mathrm{mL}, 0.5 \mathrm{~mL})$ was added to dialysis tubing cellulose membrane with a cut off molecular weight of 14,000 . The dialysis bags were dialyzed in DI water $(2 \mathrm{~mL})$ under $808 \mathrm{~nm}$ laser exposure $\left(1 \mathrm{~W} / \mathrm{cm}^{2}\right)$ for $10 \mathrm{~min}$ and the incubation medium were collected at predetermined time points. The amounts of released drug were quantified using LC-MS method. All experiments were performed in triplicate.

\subsubsection{Cell Culture}

Hep55.1c (RRID: CVCL_5766) mouse liver cancer cell line was donated by China Medical University, Taiwan. Hep55.1c was maintained in DMEM medium supplemented with $10 \%$ fetal bovine serum (FBS) and 1\% penicillin-streptomycin. Bone marrow cells which were extracted from thigh bones of 6-8 weeks C57BL/6 WT mice were induced to differentiate to DCs in 5 to 6 days culture in RPMI 1640 medium supplemented with $10 \%$ FBS, $1 \%$ sodium pyruvate, $1 \%$ nonessential amino acids, $1 \%$ penicillin/streptomycin, GM-CSF $(20 \mathrm{ng} / \mathrm{mL})$, and IL-4 $(10 \mathrm{ng} / \mathrm{mL})$. The cells were cultured in complete medium at $37^{\circ} \mathrm{C}$ in a humidified atmosphere with $5 \%$ carbon dioxide in air.

\subsubsection{Determination of Targeting Efficiency of AP3-Auncs-Anti-PDL1}

In order to observe the target behavior between AP3-AuNCs-anti-PDL1 and Hep55.1c cells, fluorescent staining technique was used to demonstrate AuNCs targeting. The fixed Hep55.1c cells' F-actin structure and nuclei were stained by green fluorescent phalloidin and blue fluorescent 4',6-diamidino-2-phenylindole (DAPI), respectively. Rhodamine B (RhoB) was conjugated with AuNCs that made nanocarriers highly visible under a fluorescence microscopy. A total amount of $3 \times 10^{5}$ Hep55.1c cells were seeded and grew on glass coverslips in the 6-well plates for $24 \mathrm{~h}$. The cells were then incubated with RhoB-labeled AP3-AuNCs or AP3-AuNCs-anti-PDL1 for different periods of time. Afterward, Hep55.1c cells were washed with PBS three times and fixed for $30 \mathrm{~min}$ with $3.7 \%$ formaldehyde. Cellular permeability was performed with $0.25 \%$ Triton X-100 for $15 \mathrm{~min}$. Finally, after cell staining with F-actin and DAPI for $1 \mathrm{~h}$ and $30 \mathrm{~min}$, respectively, the cells were mounted on a clean glass slide by mounting medium and observed under fluorescence or confocal microscopy (D-eclipse C1; Eclipse, TE2000-U, Nikon, Tokyo, Japan).

\subsubsection{Cytotoxicity Assay of Hep55.1c Treated with Photothermal Therapy}

A total of $1 \times 10^{5}$ Hep55.1c cells were cultured in a 96-well plate for $24 \mathrm{~h}$ prior to the cytotoxic test. AP3-AuNCs $(50 \mu \mathrm{g} / \mathrm{mL})$ were added to the well and incubated for $24 \mathrm{~h}$. After that, the Hep55.1c cells were treated with $808 \mathrm{~nm}$ diode laser $\left(1 \mathrm{~W} / \mathrm{cm}^{2} ; 2\right.$, 
5, 10, $15 \mathrm{~min}$ ) or nothing as control. After the treatment, the cells were maintained for further 1 day. Then, the MTS reagent (10\%) containing medium was added to each well for $2 \mathrm{~h}$ and incubation, and the optical absorbance was measured at $490 \mathrm{~nm}$ using an ELISA reader (DV990BV4, GDV, Milan, Italy). On the other hand, to evaluate the AP3-AuNCs cell cytotoxicity, after $1 \times 10^{5}$ Hep55.1c cells were cultured in a 96 well plate for $24 \mathrm{~h}$, the cells were incubated with FBS-free medium containing various concentrations of AuNCs $(0,1,10,20,40,80,100 \mu \mathrm{g} / \mathrm{mL})$ for $24 \mathrm{~h}$. Then, the MTS reagent $(10 \%)$ containing medium was added to each well and incubated for $2 \mathrm{~h}$ and the optical absorbance was measured at $490 \mathrm{~nm}$ using an ELISA reader (DV990BV4, GDV, Milan, Italy).

\subsubsection{Analysis of Mature Dcs Surface-Marker Expression}

The method refers to the protocol paper of Alka et al. [28]. After 6 days of incubation, non-adherent primary bone marrow dendritic cells (BMDC) were collected by gently pipetting them with culture medium. For in vitro DCs stimulation experiments, we cocultured AP3, AuNCs, and AP3-AuNCs (AuNCs $=50 \mu \mathrm{g} / \mathrm{mL}, \mathrm{AP3}=1 \mu \mathrm{g} / \mathrm{mL}$ ) with 2 $\times 10^{5}$ bone-derived DCs from C57BL/6 mice for $20 \mathrm{~h}$. For transwell (Corning Costar ${ }^{\circledR}$, New York, NY, USA) experiments, residues of Hep55.1c cells after photothermic treatment with AP3-AuNCs were also added to the $10^{6}$ DCs culture for $20 \mathrm{~h}$. The DCs stained with anti-CD11c-FITC (RUO), anti-CD80-PE (16-10A1), and anti-CD86-PE (GL1) were assessed by flow cytometry (Novocyte ${ }^{\circledR}$, ACEA biosciences, San Diego, CA, USA).

\section{Result and Discussion}

\subsection{Synthesis of Silver Nanocubes (Agncs) and Gold Nanocages (Auncs)}

AuNCs were prepared according to a robust protocol through two steps [24]: sulfidemediated silver nucleation and the subsequent reconstruction by the galvanic replacement reaction as the following equation (Equation (4)).

$$
3 \mathrm{Ag}_{(\mathrm{s})}+\mathrm{AuCl}^{4-}{ }_{(\mathrm{aq})} \rightarrow 3 \mathrm{Ag}^{+}{ }_{(\mathrm{aq})}+\mathrm{Au}_{(\mathrm{s})}+4 \mathrm{Cl}^{-}{ }_{(\mathrm{aq})}
$$

The size of the obtained AgNCs was observed to be around $50 \mathrm{~nm}$ in edge length measured using SEM in Figure 1a. Subsequently, AuNCs were formed in a galvanic replacement reaction and the original square shape was retained as shown in Figure 1b. A high-magnification STEM image displayed a hollow structre with porous walls in Figure 1c. During the galvanic replacement reaction, with increasing the volume of $\mathrm{HAuCl}_{4}$ solution, the absorbance peak alternated from $440 \mathrm{~nm}$ to the near infrared (NIR) range as shown in Figure 1d, which can be evidenced and monitored through serial color changes using UV-visible spectroscopy (Figure S1).

\subsection{Characterization of Ansamitocin P3-Loaded Gold Nanocages (AP3-Auncs)}

A photo-sensitive AP3-AuNCs was enshrouded with L-cysteine (CYS) through goldsulfur interaction to conjugate the biotin to bind the anti-PDL1. The solubility of AP3 is quite low (water solubility $=20 \mu \mathrm{g} / \mathrm{mL}$ ) in aqueous medium but ethanol is a better solvent for dissolving AP3 $(1 \mathrm{mg} / \mathrm{mL})$. Therefore, the AP3-AuNCs synthesized in distilled deionized water $(\mathrm{ddw}), 80 \%$ ethanol $(\mathrm{EtOH})$ solution, and 99\% EtOH were investigated $[29,30]$.

For verifying the Ansamitocin P3 loading, Fourier transform infrared spectra (FTIR) were used to verify the components of AP3-AuNCs conducted in the three solvents as shown in Figure 2. The AuNCs showed the major characteristic peak at $1647 \mathrm{~cm}^{-1}(\mathrm{C}=\mathrm{O}$ vibration) and $1385 \mathrm{~cm}^{-1}$ (C-N vibration) because of the existence of the PVP on the surface of AuNCs with its skeletal formula shown in Figure S2a. The spectrum of L-cysteine (CYS) presented a broadband at $3029 \mathrm{~cm}^{-1}\left(\mathrm{NH}_{3}{ }^{+}\right.$vibration), a peak at $2591 \mathrm{~cm}^{-1}(\mathrm{~S}-\mathrm{H}$ vibration), two peaks overlapped in $1624-1587 \mathrm{~cm}^{-1}\left(\mathrm{C}=\mathrm{O}\right.$ vibration and $\mathrm{NH}_{3}{ }^{+}$vibration), and a peak at $1490 \mathrm{~cm}^{-1}\left(\mathrm{NH}_{3}{ }^{+}\right.$bending) [31]. However, the spectra of the AP3-AuNCs did not present the major peaks of CYS corresponding to $\mathrm{NH}^{+}$vibration, $\mathrm{S}-\mathrm{H}$ vibration, and $\mathrm{NH}^{+}$bending possibly because of the gold-thiol interaction with AuNCs. The major peaks of AP3 indexed around $1594 \mathrm{~cm}^{-1}$ and $1402 \mathrm{~cm}^{-1}$ in Figure 2 appeared in all the 
samples. However, AP3-AuNCs synthesized in $80 \% \mathrm{EtOH}$ exhibited the stronger AP3 peaks. In addition, the AP3-AuNCs in $80 \% \mathrm{EtOH}$ displayed the stable morphology with a particle size distribution of approximately $120 \mathrm{~nm}$ and weak negative zeta potential of $-9.46 \mathrm{mV}$ (Table S1). Therefore, AP3-AuNCs conducted in $80 \% \mathrm{EtOH}$ solution was selected for further investigation.
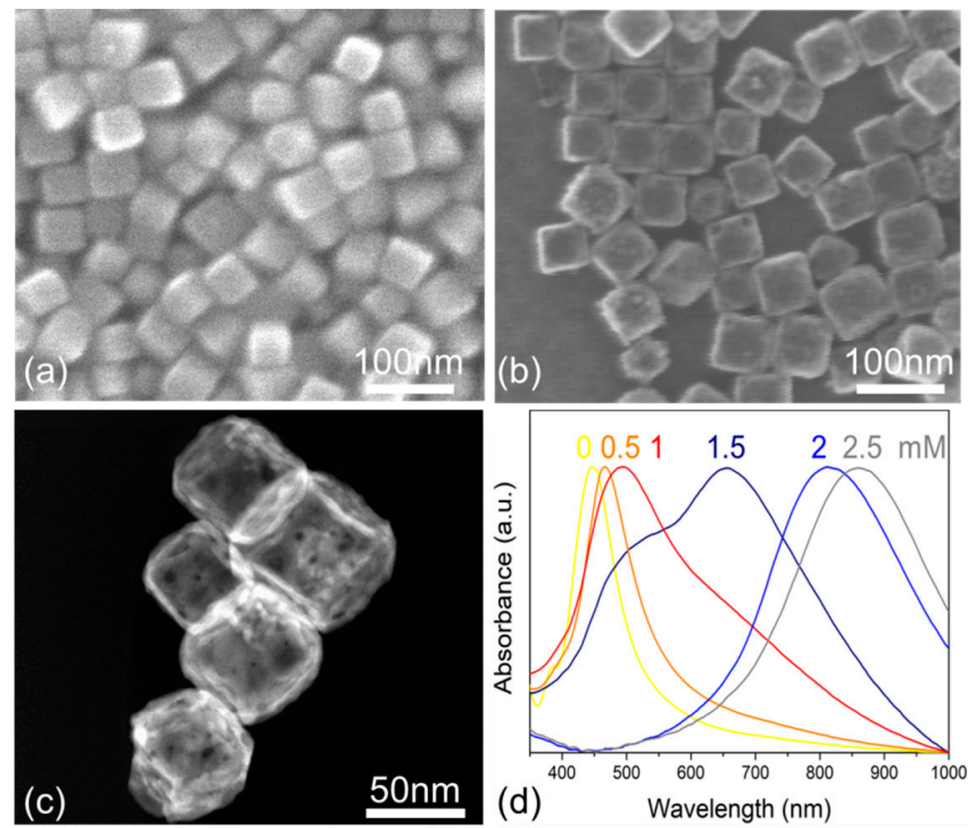

Figure 1. Images and optical properties of AgNCs and AuNCs. (a) SEM image of AgNCs, which showed approximately $50 \mathrm{~nm}$ in edge length. (b) SEM image and (c) TEM image of AuNCs showing a hollow and porous structure. (d) UV-visible-NIR absorbance spectra of AgNCs and AuNCs by adding different volumes of $\mathrm{HAuCl}_{4}$ solution $(0,0.5,1,1.5,2,2.5 \mathrm{mM})$.

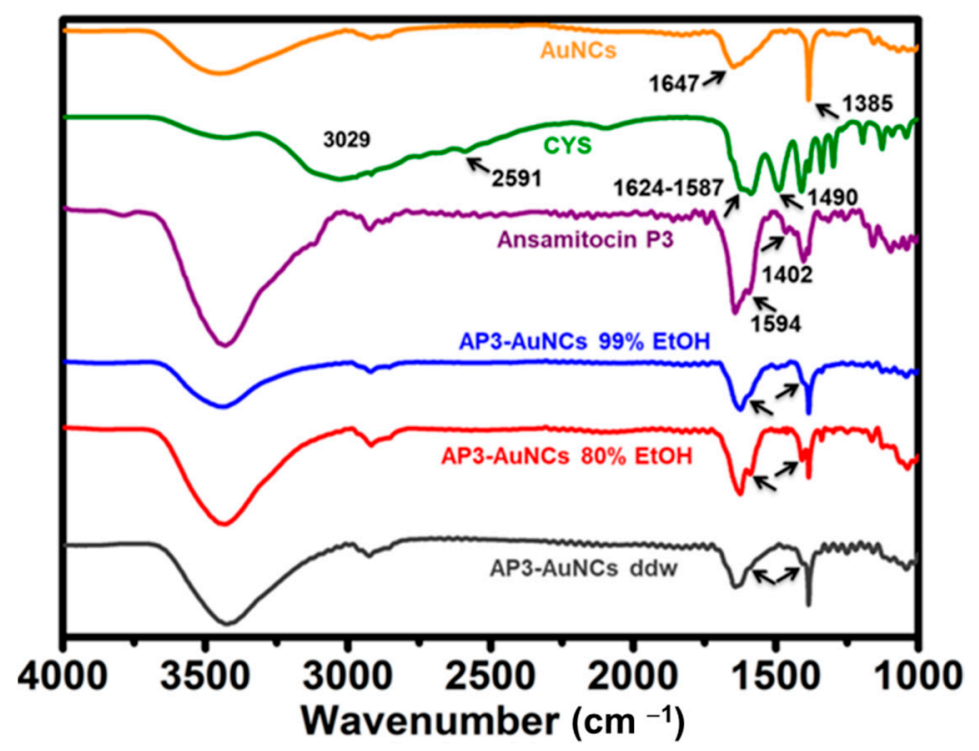

Figure 2. Fourier transform infrared spectra (FTIR) of AuNCs, CYS, AP3, AP3-AuNCs in ddw, 80\% $\mathrm{EtOH}$ and $99 \% \mathrm{EtOH}$.

To confirm the morphology of AP3-AuNCs, TEM with elemental mapping of AP3AuNCs using EDS was shown in Figure 3. The AuNCs were completely covered by the CYS to reduce AP3 leakage in Figure 3a. Further, the AuNCs mapping (Figure 3b) showed 
a clear hollow and square structure. The elemental mapping of sulfur (S) and nitrogen $(\mathrm{N})$ overlapped the area of Au in Figure 3c-e was attributed to the AuNCs with the CYS coated. Moreover, the chlorine $(\mathrm{Cl})$ in Figure $3 \mathrm{f}$ indicated the AP3 drug distributed in the inner layer of AuNCs. From the results, it can be confirmed that the AP3 was successfully loaded in the AuNCs and the AuNCs was covered by the CYS to reduce the leakage of AP3. The encapsulation efficiency (EE) and loading capacity ( $\mu \mathrm{g}$ AP3/ $\mu \mathrm{g}$ AP3-AuNCs) of AP3 were measured around $49 \%$ and $2.04 \%$, respectively.
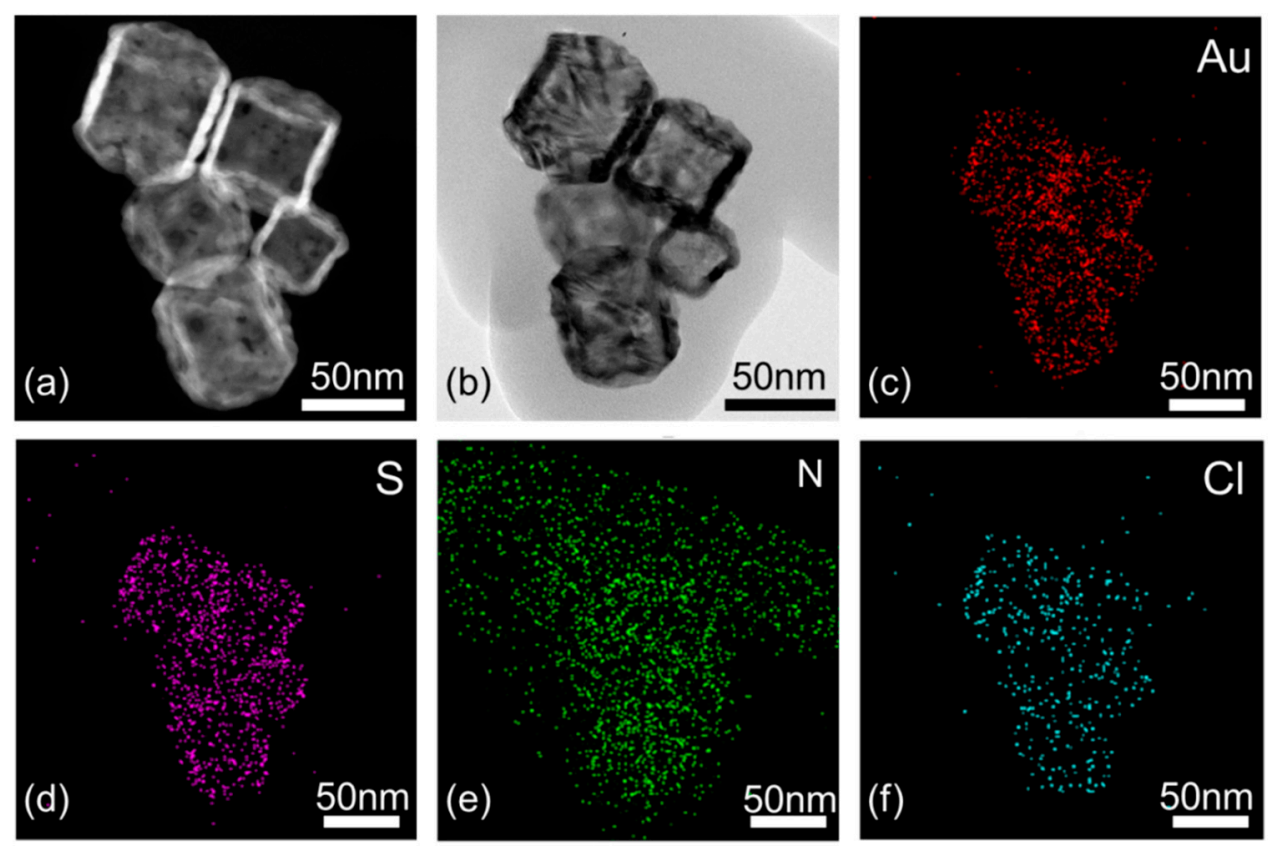

Figure 3. (a) STEM image, (b) TEM image and (c-f) elemental mapping of Au, S, N, and Cl AP3AuNCs.

\subsection{Heating Efficiency and AP3 Release of AP3-Auncs}

As a photothermal treatment was applied to induce death of heat-sensitive cancer cells, AP3 was simultaneously released outside the tumor cell to activate the immature DCs in the tumor microenvironment. To evaluate the photothermal effect of AP3-AuNCs, the temperature increase of AP3-AuNCs with irradiation time under $808 \mathrm{~nm}$ laser irradiation at a power density of $1 \mathrm{~W} / \mathrm{cm}^{2}$ was monitored. As shown in Figure $4 a$, AuNCs and AP3AuNCs displayed significant temperature increase to $43^{\circ} \mathrm{C}$ in $150 \mathrm{~s}$ and $180 \mathrm{~s}$, respectively, indicating that the heating efficiency of both AuNCs and AP3-AuNCs was barely influenced by CYS coating and AP3 loading. In contrast, only a slight temperature increase from $25^{\circ} \mathrm{C}$ to $29^{\circ} \mathrm{C}$ was observed from the control of ddw. The photothermal stability of AP3-AuNCs and AuNCs was further investigated. As shown in Figure S3, the morphology of both nanoparticles still presented a cubic shape after the samples were repeated three times with a total irradiation time of $45 \mathrm{~min}$. In addition, the PTCE of AuNCs and AP3-AuNCs were calculated about $65.19 \%$ and $58.54 \%$, respectively. The results showed that the PTCE of both AuNCs and AP3-AuNCs was higher than gold nanorods (GNRs) and organic nanoparticle under the $808 \mathrm{~nm}$-laser. This illustrated that AP3-AuNCs can facilitate the photothermal ablation of cancer cells [32,33]. Further, AP3 drug release from AP3-AuNCs can be further triggered and promoted by using $808 \mathrm{~nm}$ laser irradiation at $1 \mathrm{~W} / \mathrm{cm}^{2}$. The results indicated that AP3-AuNCs presented a sustained AP3 release with time and reached up $90 \%$ in 10 min in Figure 4b. In contrast, the AP3 release was negligible without NIR triggering in control group, showing the AP3 remaining in AuNCs at room temperature. 
(a)

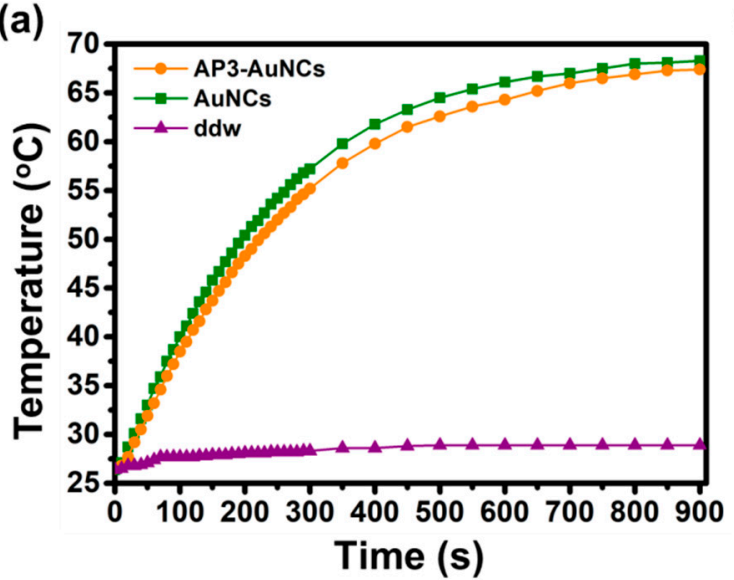

(b)

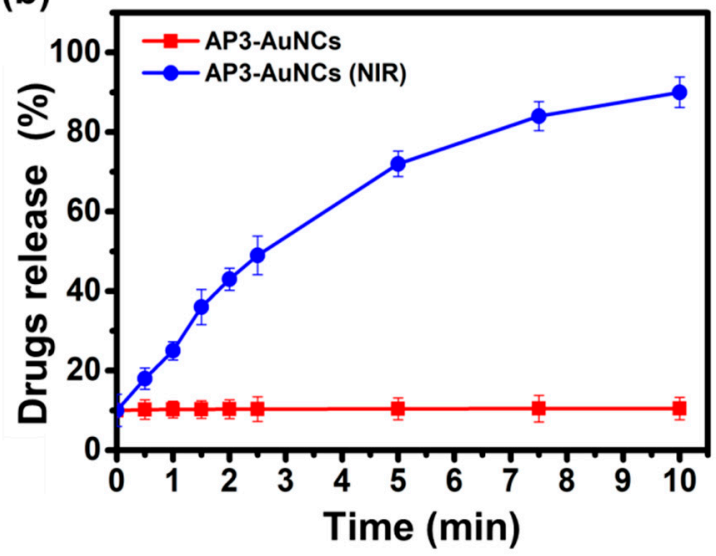

Figure 4. (a) Profile of temperature increase for $50 \mu \mathrm{g} / \mathrm{mL}$ AuNCs and AP3-AuNCs upon $808 \mathrm{~nm}$ laser irradiation at a power density of $1 \mathrm{~W} / \mathrm{cm}^{2}$. (b) Cumulative AP3 release from AP3-AuNCs with NIR irradiation time.

\subsection{Characteristic of Anti-PDL1-Targeted AP3-Auncs}

One of the important concerns of cancer therapy is to selectively deliver therapeutic agents to the targeted cells. Programmed death-ligand 1 (PDL1) has been exclusively overexpressed on cell surface of numerous metastatic cancer cells so it can also be considered as a reliable surface-binding site for targeted therapy to increase the activities of immune responses [34,35]. As a result, the surface of AuNCs was grafted with CYS-biotin to conjugate the anti-PDL1. To ensure the conjugation and direction of anti-PDL1 to present its targeting function, the immunofluorescence for targeting the fragment of $F\left(a b^{\prime}\right) 2$ was performed in Figure 5 which confirmed that the anti-PDL1 has been successfully bonded on the AuNCs surface via Biotin. In addition, the direction of the anti-PDL1 Fab is outward to prove its specific function to tumor cells.

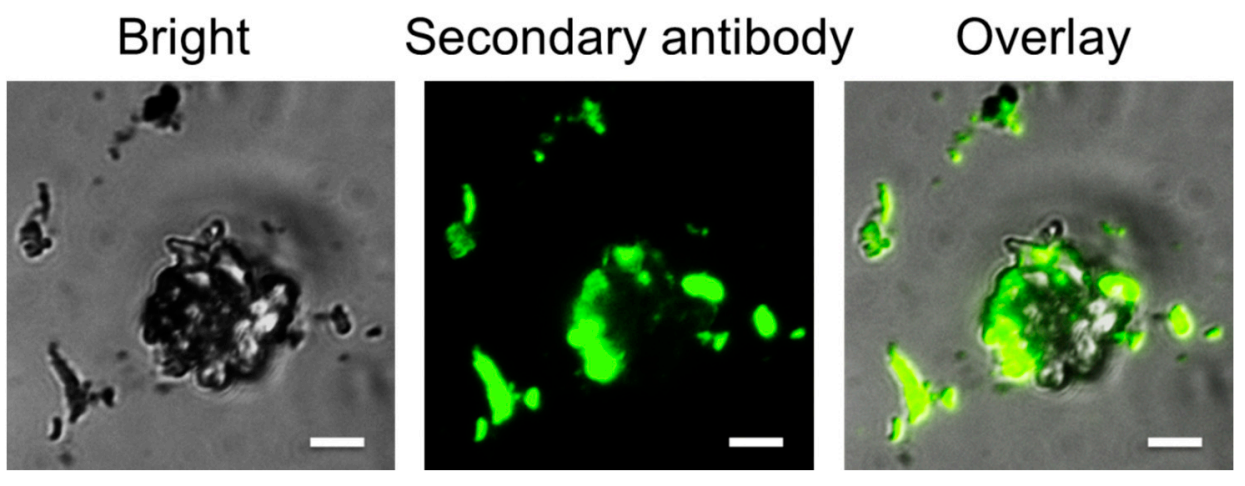

Figure 5. Immunofluorescence of AP3-AuNCs-anti-PDL1. (Scale bar $=1 \mu \mathrm{m}$ ).

\subsection{Cellular Targeting Efficiency of AP3-Auncs-Anti-PDL1}

Hepatocellular carcinoma (HCC), also known as liver cancer, exhibited high postoperative recurrence and resistance to chemotherapeutic drugs, a novel and effective therapy for HCC is thus urgently needed. The highly immunosuppressive microenvironment of HCC tumors suggested that HCC may be suitable for immunotherapy, and anti-PDL1 immune checkpoint inhibitors showed to be promising for the treatment of HCC [36-38]. To evaluate internalization of nanocages, rhodamine B (RhoB) was labeled to the AP3-AuNCs for fluorescent detection. The negligible red fluorescence was observed for the cells treated with RhoB-labeled AP3-AuNCs for $1 \mathrm{~h}$ and $4 \mathrm{~h}$ in Figure 6a. To enhance AP3-AuNCs targeting, RhoB-labeled AP3-AuNCs were conjugated with anti-PDL1 antibody. The results in Figure $6 \mathrm{~b}$ showed that the targeting efficiency of RhoB-labeled AP3-AuNCs-anti-PDL1 to HCC was significantly higher as compared to that of AP3-AuNCs without anti-PDL1 
within 1-h incubation period and more obvious after 4-h incubation in Figure $6 \mathrm{~b}$. The results demonstrated that RhoB-labeled AP3-AuNCs-anti-PDL1 could rapidly and effectively target to Hep55.1c cell at a very short time through antibody-antigen specific interaction.

(a)

$1 \mathrm{~h}$

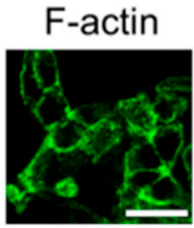

$4 \mathrm{~h}$

(b)

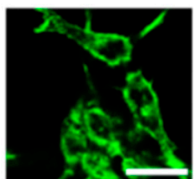

F-actin

$1 \mathrm{~h}$

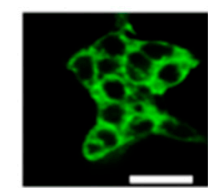

$4 \mathrm{~h}$

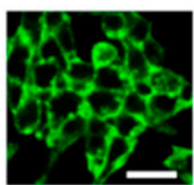

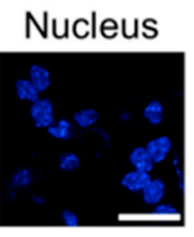

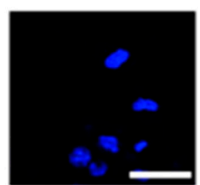

Nucleus
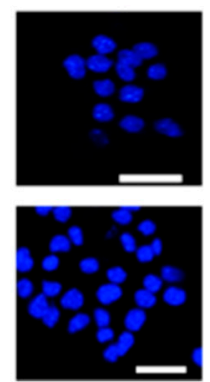
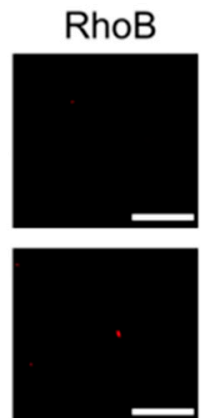

RhoB
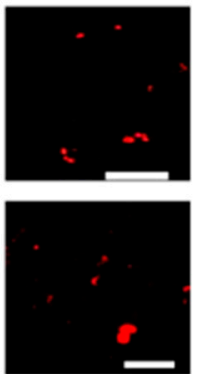
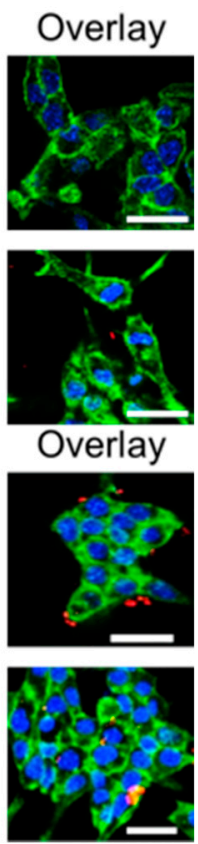

Figure 6. The cell association evaluation of (a) non-targeted RhoB-labeled AP3-AuNCs and (b) RhoBlabeled AP3-AuNCs-anti-PDL1 with $1 \mathrm{~h}$ and $4 \mathrm{~h}$ incubation using fluorescent staining. The f-actin, the nucleus of cells, and the RhoB-labeled AP3-AuNCs/RhoB-labeled AP3-AuNCs -anti-PDL1 were labeled as green, blue, and red colors, respectively (Scale bar $=10 \mu \mathrm{m}$ ).

\subsection{Cytotoxicity Assay and Photothermal Therapy of AP3-Auncs}

To investigate the cytotoxicity of AP3-AuNCs and AuNCs, Hep55.1c cells were incubated with different concentrations of AP3-AuNCs and AuNCs for $24 \mathrm{~h}$ in dark. Cell viability test in Figure S4 showed that the AuNCs and AP3-AuNCs displayed negligible cytotoxicity with the incubation of $100 \mu \mathrm{g} / \mathrm{mL}$ AuNCs, indicating that both materials displayed good biocompatibility under proper dose utilization. When the heating temperature was controlled at $41-45^{\circ} \mathrm{C}$, nanoparticle-induced hyperthermia can lead to tumor cell death without causing serious injury to adjoining normal tissues. The phototherapeutic capabilities of AuNCs and AP3-AuNCs to Hep55.1c cells were further studied in dark or under $808 \mathrm{~nm}$ laser irradiation $\left(1 \mathrm{~W} / \mathrm{cm}^{2}\right)$. The cell viabilities of Hep55.1c cells were not significantly affected by AuNCs and AP3-AuNCs (concentration $=50 \mu \mathrm{g} / \mathrm{mL}$ ) under dark condition in Figure 7. When the Hep55.1c cells were treated with NIR for $2 \mathrm{~min}$, the cell viabilities were between 75 and $80 \%$ because of poor thermal efficiency. However, as the Hep55.1c cells were treated for 5, 10 and $15 \mathrm{~min}$, the cell viabilities were reduced to below $50 \%$. In contrast, minimal effect was observed without AP3-AuNCs even though the cells were irradiated under $808 \mathrm{~nm}$ for $10 \mathrm{~min}$. These results demonstrated that AuNCs displayed excellent photothermic effect under a low-power density of $1 \mathrm{~W} / \mathrm{cm}^{2}$ at a lowconcentration of AuNCs compared to Au-nanostructured nanoparticles [39-41]. More importantly, both AP3-AuNCs and AuNCs displayed similar cell viabilities, proving that AuNCs with AP3 loaded showed no interference for NIR photothermic effect. 


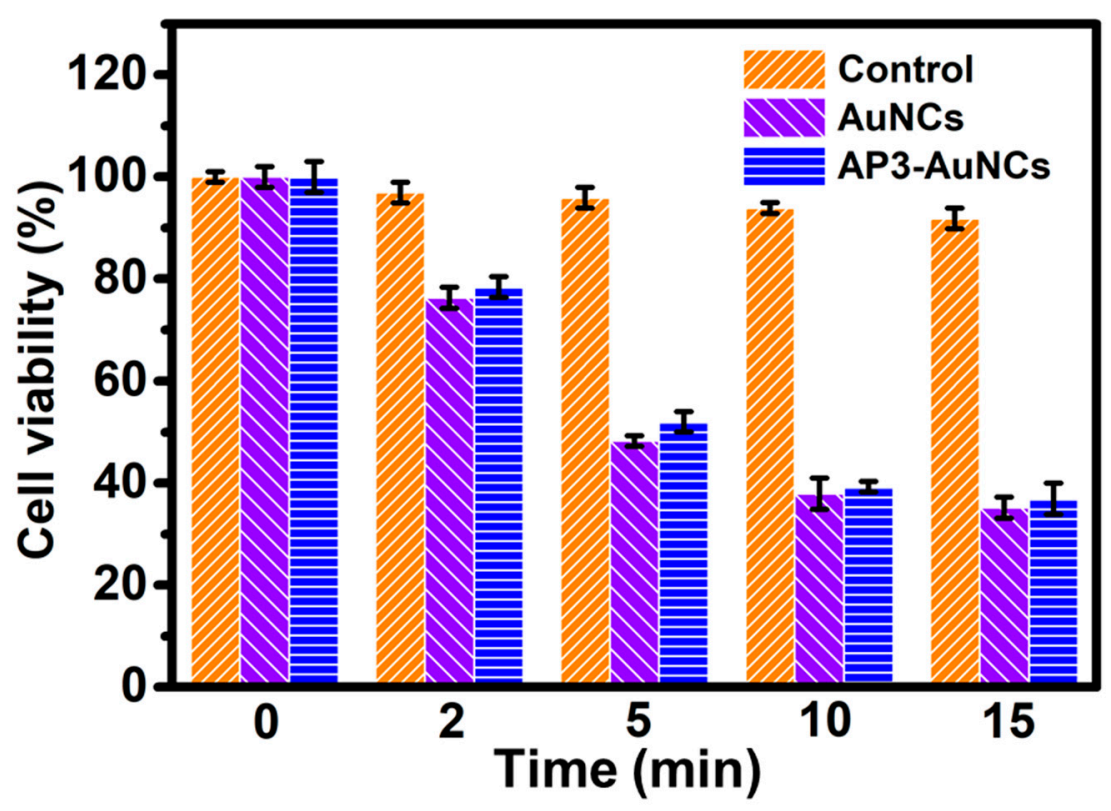

Figure 7. In vitro cell viability for AP3-AuNCs and AuNCs post $24 \mathrm{~h}$ incubation induced by PTT. For PTT effect, the cells were irradiated under $808 \mathrm{~nm}$ diode laser $\left(1 \mathrm{~W} / \mathrm{cm}^{2}\right)$ for 2, 5, 10, and $15 \mathrm{~min}$. Data are expressed as mean $\pm \mathrm{SD}, n=3$.

\subsection{In Vitro Dcs Maturation Efficiency of AP3-Auncs}

For efficient T-cell stimulation, mature DCs should express or upregulate T-cellcostimulatory molecules. $\mathrm{CD}_{11 \mathrm{c}^{+}}$is transmembrane protein that is expressed on the immature DCs and mature DCs. Mature DCs could express up-regulation of co-stimulatory molecules (CD80 and CD86) on the surface. As shown in Figure 8, DCs maturation induced by AP3 and PTT was further assessed for the untreated Hep55.1c cells added with AuNCs, AP3 and AP3-AuNCs in the transwell systems by flow cytometry. The DCs maturation is limited for AP3 group only, but an obvious increase in DCs maturation was observed in AP3-AuNCs because a higher dosage of AP3 could be delivered to the DCs using AuNCs. On the other hand, as the Hep55.1c were treated by PTT using AuNCs or AuNCs-AP3, the AP3-AuNCs+NIR-10 min exhibited the highly activated DC maturation with 2-2.5 fold higher than AP3-AuNCs or control+NIR. Further, to evaluate the combination of AP3 dose and TAAs, NIR treatment with various time was applied. Figure $8 \mathrm{~b}$ showed that CD80 was measured as $55.98 \pm 1.38,66.54 \pm 0.9$, and $81.58 \pm 1.58 \%$ under PTT treatment for 2 , 5 , and 10 min of PTT, respectively. In addition, CD86 was also measured as $54.81 \pm 2.12$, $63.38 \pm 0.7$, and $73.85 \pm 1.61 \%$ in 2, 5, and $10 \mathrm{~min}$ of PTT in Figure 8d, respectively. As compared to DCs maturation in Figure 8 with the temperature profile of AP3-AuNCs and cumulative AP3 release upon $808 \mathrm{~nm}$ laser irradiation at a power density of $1 \mathrm{~W} / \mathrm{cm}^{2}$ in Figure 4, it was noted that the AP3-AuNCs was irradiated with NIR for $2 \mathrm{~min}$, the temperature up to $43-45^{\circ} \mathrm{C}$ is enough for killing the tumor cells (20\% in Figure 7) to produce TAAs but AP3 release is about $40-45 \%$. With increasing exposure time to $10 \mathrm{~min}$ at PTT, AP3-AuNCs+NIR-10 min exhibited the activated DC maturation with a $\sim 30 \%$ increase compared to AP3-AuNCs+NIR-2min, which can be attributed to the significant release of AP3 to $90 \%$. The results illustrated the importance of significant AP3 release in triggering the effective DCs maturation and then T-cell activation under the combination treatment of post-PTT and AP3. Compared with photothermal or photodynamic nanoparticle carrying immune-adjuvant agent [18,42], AP3-AuNCs displayed high DCs maturation in vitro, which can be attributed to the synergistic effect of generating tumor-associated antigens and simultaneously releasing AP3 to stimulate DCs. 
(a)
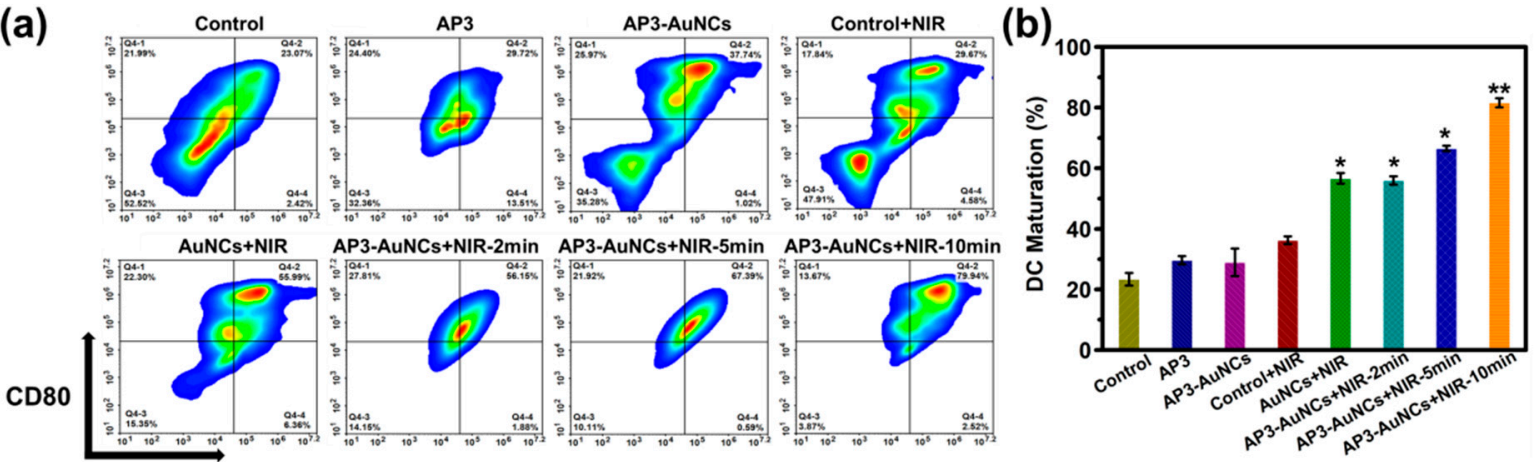

(c)

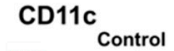

AP3
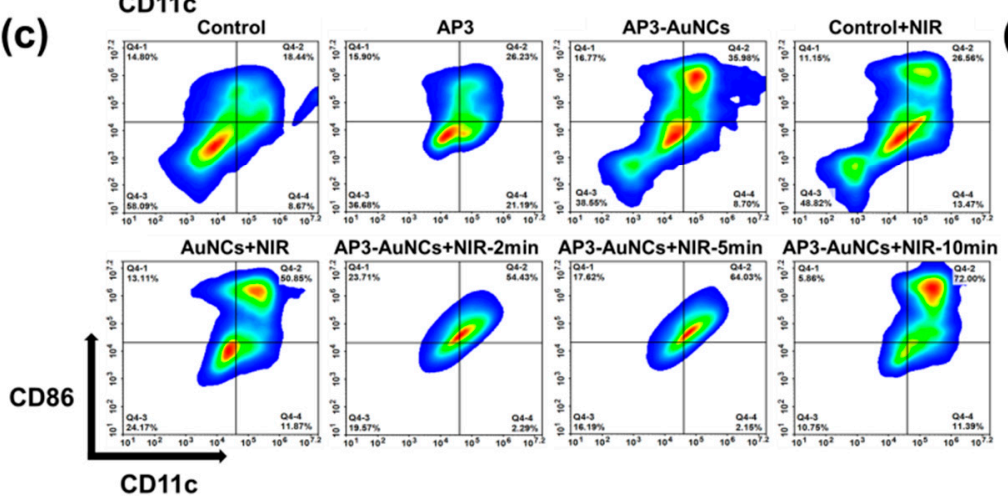

(d)

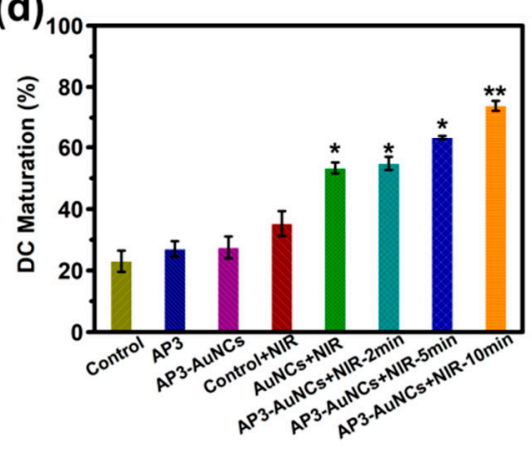

Figure 8. Flow cytometry expression of (a-b) CD11c CD80 (c-d) CD11c CD86 on mature DCs surface after feeding AP3, AuNCs and AP3-AuNCs. Two-tail Student's test was used. ${ }^{*} p<0.05,{ }^{* *} p<0.01$.

\section{Conclusions}

In this study, a multifunctional gold nanocages (AuNCs)-based nanocarrier was developed for hepatocellular carcinoma (HCC) by nanoparticle-assisted photothermal therapy (PTT), chemotherapeutic agent-triggered dendritic cells (DCs) activation, and checkpoint immune therapy. By using this gold nanocages (AuNCs)-based nanocarrier, the hydrophobic AP3 can be closely encapsulated to promote the release as a potent inducer of phenotypic and functional maturation of DCs under the NIR exposure to significantly trigger the maturation of DCs and immunity promotion. This AuNCs-based nanocarrier with the anti-PDL1 as a platform to produce the TAAs and control the AP3 release for stimulating DCs maturation, leading to the activation of $\mathrm{T}$ cell and specific targeting of the tumor cells to create an immunogenic tumor microenvironment. Moreover, the achievement illustrates the promising in HCC cancer therapy using AP3-AuNCs-anti-PDL1 in vivo and the clinical applications.

Supplementary Materials: The following are available online at https:/ / www.mdpi.com/article/10 .3390 / polym13162726/s1, Figure S1: HAuCl 4 solution $(0,0.5,1,1.5,2,2.5 \mathrm{mM})$ were used to titrate Ag nanocubes suspension and brilliant color was changed during Ag Aualloy formation; Figure S2: The structure of PVP and Anasmitocin P3; Figure S3: The morphology of AuNCs and AP3 AuNCs under $808 \mathrm{~nm}$ irradiation; Figure S4: Evaluation of cell viability of Hep55.1c cells after incubation with various concentrations of AuNCs and AP3-AuNCs in dark for $24 \mathrm{~h}$, respectively. Error bars are expressed standard error with $n=3$; Table S1: Characterization of the AP3-AuNC constructed in different solvents.

Author Contributions: Conceptualization, H.-W.C. and Y.-L.O.; methodology, Y.-L.O.; validation, Y.-L.O., C.-C.K. and H.-Y.T.; formal analysis, H.-W.C., C.-C.K. and H.-Y.T.; investigation, Y.-L.O. and C.-C.K.; resources, H.-E.L.; data curation, H.-W.C. and Y.-L.O.; writing-original draft preparation, H.-W.C.; writing—review and editing, H.-E.L.; visualization, H.-W.C. and H.-Y.T.; supervision, H.E.L.; project administration, H.-E.L.; funding acquisition, H.-E.L. All authors have read and agreed to the published version of the manuscript. 
Funding: The authors thank for the research grants of MOST 110-2224-E-080-001- and MOST 1092224-E-080-001- from the Ministry of Science and Technology in Taiwan.

Institutional Review Board Statement: Not applicable.

Informed Consent Statement: Not applicable.

Data Availability Statement: The data presented in this study are available on request from the corresponding author.

Conflicts of Interest: There are no conflicts of interest to declare.

\section{References}

1. Darvin, P.; Toor, S.M.; Nair, V.S.; Elkord, E. Immune checkpoint inhibitors: Recent progress and potential biomarkers. Exp. Mol. Med. 2018, 50, 1-11. [CrossRef]

2. Khalil, D.N.; Smith, E.L.; Brentjens, R.J.; Wolchok, J.D. The future of cancer treatment: Immunomodulation, CARs and combination immunotherapy. Nat. Rev. Clin. Oncol. 2016, 13, 273-290. [CrossRef] [PubMed]

3. Palucka, K.; Banchereau, J. Dendritic-Cell-Based Therapeutic Cancer Vaccines. Immunity 2013, 39, 38-48. [CrossRef]

4. Rosenberg, S.A.; Restifo, N.P. Adoptive cell transfer as personalized immunotherapy for human cancer. Science 2015, 348, 62-68. [CrossRef] [PubMed]

5. Scotti, C.; Iamele, L.; Vecchia, L. Antibody-Drug conjugates: Targeted weapons against cancer. Antib. Technol. J. 2015, 5, 1-13. [CrossRef]

6. Johnston, M.P.; Khakoo, S.I. Immunotherapy for hepatocellular carcinoma: Current and future. World J. Gastroenterol. 2019, 25, 2977-2989. [CrossRef]

7. Robert, C.; Schachter, J.; Long, G.V.; Arance, A.; Grob, J.J.; Mortier, L.; Daud, A.; Carlino, M.S.; McNeil, C.; Lotem, M.; et al. Pembrolizumab versus Ipilimumab in Advanced Melanoma. N. Engl. J. Med. 2015, 372, 2521-2532. [CrossRef]

8. Keenan, B.P.; Fong, L.; Kelley, R.K. Immunotherapy in hepatocellular carcinoma: The complex interface between inflammation, fibrosis, and the immune response. J. Immunother. Cancer 2019, 7, 267. [CrossRef]

9. Chen, D.S.; Mellman, I. Oncology Meets Immunology: The Cancer-Immunity Cycle. Immunity 2013, 39, 1-10. [CrossRef] [PubMed]

10. Zhou, J.; Wang, G.; Chen, Y.; Wang, H.; Hua, Y.; Cai, Z. Immunogenic cell death in cancer therapy: Present and emerging inducers. J. Cell. Mol. Med. 2019, 23, 4854-4865. [CrossRef]

11. Kroemer, G.; Galluzzi, L.; Kepp, O.; Zitvogel, L. Immunogenic Cell Death in Cancer Therapy. Annu. Rev. Immunol. 2013, 31, 51-72. [CrossRef]

12. Toraya-Brown, S.; Fiering, S. Local tumour hyperthermia as immunotherapy for metastatic cancer. Int. J. Hyperth. 2014, 30, 531-539. [CrossRef]

13. Slovak, R.; Ludwig, J.M.; Gettinger, S.N.; Herbst, R.S.; Kim, H.S. Immuno-Thermal ablations-Boosting the anticancer immune response. J. Immunother. Cancer 2017, 5, 78. [CrossRef] [PubMed]

14. Yu, J.; Liu, S.; Wang, Y.; He, X.; Zhang, Q.; Qi, Y.; Zhou, D.; Xie, Z.; Li, X.; Huang, Y. Synergistic enhancement of immunological responses triggered by hyperthermia sensitive Pt NPs via NIR laser to inhibit cancer relapse and metastasis. Bioact. Mater. 2021. [CrossRef]

15. Liu, Q.; Zhai, B.; Yang, W.; Yu, L.-X.; Dong, W.; He, Y.-Q.; Chen, L.; Tang, L.; Lin, Y.; Huang, D.-D.; et al. Abrogation of Local Cancer Recurrence After Radiofrequency Ablation by Dendritic Cell-based Hyper-thermic Tumor Vaccine. Mol. Ther. 2009, 17, 2049-2057. [CrossRef] [PubMed]

16. Xu, P.; Liang, F. Nanomaterial-Based Tumor Photothermal Immunotherapy. Int. J. Nanomed. 2020, 15, 9159-9180. [CrossRef]

17. Xu, L.; Zhang, W.; Park, H.-B.; Kwak, M.; Oh, J.; Lee, P.C.W.; Jin, J.-O. Indocyanine green and poly I:C containing ther-moresponsive liposomes used in immune-photothermal therapy prevent cancer growth and metastasis. J. Immunother. Cancer 2019, 7, 220. [CrossRef]

18. Chen, Q.; Xu, L.; Liang, C.; Wang, C.; Peng, R.; Liu, Z. Photothermal therapy with immune-adjuvant nanoparticles together with checkpoint blockade for effective cancer immunotherapy. Nat. Commun. 2016, 7, 13193. [CrossRef] [PubMed]

19. Zhao, X.; Yang, K.; Zhao, R.; Ji, T.; Wang, X.; Yang, X.; Zhang, Y.; Cheng, K.; Liu, S.; Hao, J.; et al. Inducing enhanced immunogenic cell death with nanocarrier-based drug delivery systems for pancreatic cancer therapy. Biomater. 2016, 102, 187-197. [CrossRef]

20. Martin, K.; Müller, P.; Schreiner, J.; Prince, S.S.; Lardinois, D.; Heinzelmann-Schwarz, V.; Thommen, D.S.; Zippelius, A. The microtubule-depolymerizing agent ansamitocin P3 programs dendritic cells toward enhanced anti-tumor immunity. Cancer Immunol. Immunother. 2014, 63, 925-938. [CrossRef]

21. Qian, X.; Peng, X.-H.; Ansari, D.O.; Yin-Goen, Q.; Chen, G.Z.; Shin, D.M.; Yang, L.; Young, A.N.; Wang, M.D.; Nie, S. In vivo tumor targeting and spectroscopic detection with surface-enhanced Raman nanoparticle tags. Nat. Biotechnol. 2007, 26, 83-90. [CrossRef] [PubMed]

22. Li, W.; Cai, X.; Kim, C.; Sun, G.; Zhang, Y.S.; Deng, R.; Yang, M.; Chen, J.; Achilefu, S.; Wang, L.; et al. Gold nanocages covered with thermally-responsive polymers for controlled release by high-intensity focused ultrasound. Nanoscale 2011, 3, 1724-1730. [CrossRef] 
23. Yang, X.; Yang, M.; Pang, B.; Vara, M.; Xia, Y. Gold Nanomaterials at Work in Biomedicine. Chem. Rev. 2015, 115, 10410-10488. [CrossRef] [PubMed]

24. Skrabalak, S.E.; Au, L.; Li, X.; Xia, Y. Facile synthesis of Ag nanocubes and Au nanocages. Nat. Protoc. 2007, 2, 2182-2190. [CrossRef]

25. Roper, D.K.; Ahn, W.; Hoepfner, M. Microscale Heat Transfer Transduced by Surface Plasmon Resonant Gold Nanoparticles. J. Phys. Chem. C 2007, 111, 3636-3641. [CrossRef] [PubMed]

26. Shao, W.; Wei, Q.; Wang, S.; Li, F.; Wu, J.; Ren, J.; Cao, F.; Liao, H.; Gao, J.-Q.; Zhou, M.; et al. Molecular engineering of D-A-D conjugated small molecule nanoparticles for high performance NIR-II photothermal therapy. Mater. Horizons 2020, 7, 1379-1386. [CrossRef]

27. Iwamoto, N.; Shimomura, A.; Tamura, K.; Hamada, A.; Shimada, T. LC-MS bioanalysis of trastuzumab and released em-tansine using nano-surface and molecular-orientation limited (nSMOL) proteolysis and liquid-liquid partition in plasma of trastuzumab emtansine-treated breast cancer patients. J. Pharm. Biomed. Anal. 2017, 145, 33-39. [CrossRef]

28. Madaan, A.; Verma, R.; Singh, A.T.; Jain, S.K.; Jaggi, M. A stepwise procedure for isolation of murine bone marrow and gener-ation of dendritic cells. J. Biol. Methods 2014, 1, e1. [CrossRef]

29. Ilyas, S.U.; Pendyala, R.; Marneni, N. Stability and Agglomeration of Alumina Nanoparticles in Ethanol-Water Mixtures. Procedia Eng. 2016, 148, 290-297. [CrossRef]

30. Tilaki, R.M.; Zad, A.I.; Mahdavi, S.M. The effect of liquid environment on size and aggregation of gold nanoparticles prepared by pulsed laser ablation. J. Nanoparticle Res. 2006, 9, 853-860. [CrossRef]

31. Nikolić, V.D.; Ilić, D.P.; Nikolić, L.B.; Stanković, M.Z.; Stanojević, L.P.; Savić, I.M.; Savić, I.M. The synthesis and structure char-acterization of deoxyalliin and alliin. Adv. Technol. 2012, 1, 38-46.

32. Shan, B.; Wang, H.; Li, L.; Zhou, G.; Wen, Y.; Chen, M.; Li, M. Rationally designed dual-plasmonic gold nanorod@cuprous selenide hybrid heterostructures by regioselective overgrowth for in vivo photothermal tumor ablation in the second near-infrared biowindow. Theranostics 2020, 10, 11656-11672. [CrossRef]

33. Wang, J.; Mei, T.; Liu, Y.; Zhang, Y.; Zhang, Z.; Hu, Y.; Wang, Y.; Wu, M.; Yang, C.; Zhong, X.; et al. Dual-targeted and MRI-guided photothermal therapy via iron-based nanoparticles-incorporated neutrophils. Biomater. Sci. 2021, 9, 3968-3978. [CrossRef]

34. Chiang, C.-S.; Lin, Y.-J.; Lee, R.; Lai, Y.-H.; Cheng, H.-W.; Hsieh, C.-H.; Shyu, W.-C.; Chen, S.-Y. Combination of fucoidan-based magnetic nanoparticles and immunomodulators enhances tumour-localized immunotherapy. Nat. Nanotechnol. 2018, 13, 746-754. [CrossRef]

35. Dougan, M.; Ingram, J.R.; Jeong, H.-J.; Mosaheb, M.M.; Bruck, P.T.; Ali, L.; Pishesha, N.; Blomberg, O.; Tyler, P.M.; Servos, M.M.; et al. Targeting Cytokine Therapy to the Pancreatic Tumor Microenvironment Using PD-L1-Specific VHHs. Cancer Immunol. Res. 2018, 6, 389-401. [CrossRef]

36. Calderaro, J.; Rousseau, B.; Amaddeo, G.; Mercey, M.; Charpy, C.; Costentin, C.; Luciani, A.; Zafrani, E.-S.; Laurent, A.; Azoulay, D.; et al. Programmed death ligand 1 expression in hepatocellular carcinoma: Relationship With clinical and pathological features. Hepatology 2016, 64, 2038-2046. [CrossRef]

37. Kudo, M. Immuno-Oncology in Hepatocellular Carcinoma: 2017 Update. Oncol. 2017, 93, 147-159. [CrossRef] [PubMed]

38. Liu, H.; Shen, J.; Lu, K. IL-6 and PD-L1 blockade combination inhibits hepatocellular carcinoma cancer development in mouse model. Biochem. Biophys. Res. Commun. 2017, 486, 239-244. [CrossRef]

39. Yang, S.; Yao, D.; Wang, Y.; Yang, W.; Zhang, B.; Wang, D. Enzyme-triggered self-assembly of gold nanoparticles for enhanced retention effects and photothermal therapy of prostate cancer. Chem. Commun. 2018, 54, 9841-9844. [CrossRef] [PubMed]

40. Sun, Y.; Wang, Q.; Chen, J.; Liu, L.; Ding, L.; Shen, M.; Li, J.; Han, B.; Duan, Y. Temperature-Sensitive Gold Nanoparticle-Coated Pluronic-PLL Nanoparticles for Drug Delivery and Chemo-Photothermal Therapy. Theranostics 2017, 7, 4424-4444. [CrossRef] [PubMed]

41. Ju, Y.; Zhang, H.; Yu, J.; Tong, S.; Tian, N.; Wang, Z.; Wang, X.; Su, X.; Chu, X.; Lin, J.; et al. Monodis-Perse Au-Fe2C Janus Nanoparticles: An Attractive Multifunctional Material for Triple-Modal Imaging-Guided Tumor Pho-tothermal Therapy. ACS Nano 2017, 11, 9239-9248. [CrossRef] [PubMed]

42. Xu, J.; Xu, L.; Wang, C.; Yang, R.; Zhuang, Q.; Han, X.; Dong, Z.; Zhu, W.; Peng, R.; Liu, Z. Near-Infrared-Triggered Photodynamic Therapy with Multitasking Upconversion Nanoparticles in Combination with Checkpoint Blockade for Immunotherapy of Colorectal Cancer. ACS Nano 2017, 11, 4463-4474. [CrossRef] [PubMed] 